Geometry $\&$ Topology

Volume 8 (2004) 77-113

Published: 22 January 2004

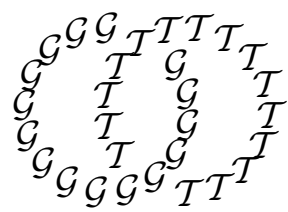

\title{
The disjoint curve property
}

\author{
SAul Schleimer \\ Department of Mathematics, UIC \\ 851 South Morgan Street \\ Chicago, Illinois 60607, USA \\ Email: saul@math.uic.edu
}

URL: http://www.math.uic.edu/^saul

\begin{abstract}
A Heegaard splitting of a closed, orientable three-manifold satisfies the disjoint curve property if the splitting surface contains an essential simple closed curve and each handlebody contains an essential disk disjoint from this curve [Thompson, 1999]. A splitting is full if it does not have the disjoint curve property. This paper shows that in a closed, orientable three-manifold all splittings of sufficiently large genus have the disjoint curve property. From this and a solution to the generalized Waldhausen conjecture it would follow that any closed, orientable three manifold contains only finitely many full splittings.
\end{abstract}

\section{AMS Classification numbers Primary: 57M99}

Secondary: $57 \mathrm{M} 27,57 \mathrm{~N} 10$

Keywords: Heegaard splittings, disjoint curve property, Waldhausen Conjecture

Proposed: Cameron Gordon

Seconded: David Gabai, Joan Birman
Received: 29 May 2002

Revised: 21 January 2004 
Saul Schleimer

\section{History and overview}

The classification of Heegaard splittings of three-manifolds is a long outstanding problem. It is natural to add restrictions to the class of three-manifolds considered. Haken's lemma [3], that all splittings of a reducible manifold are themselves reducible, can be considered one of the first results in this direction. Strong irreducibility was introduced by Casson and Gordon [2] as a generalization of irreducibility. They showed that all splittings of a non-Haken manifold are either reducible or strongly irreducible. Thompson 21 later defined the disjoint curve property (DCP) as a further generalization of reducibility. She deduced that all splittings of a toroidal three-manifold have the disjoint curve property. Hempel [5], using the classification of splittings of Seifert fibred spaces [14, has shown that each of these has the disjoint curve property. Thus, in any three-manifold which is reducible, toroidal, or a Seifert fibred space, all Heegaard splittings have the disjoint curve property.

However, it is certainly not the case that all splittings of all manifolds have the disjoint curve property. Hempel (referring to Luo) in [5] adapts an argument of Kobayashi [10] to produce examples of splittings which are full and are in fact arbitrarily far from having the DCP.

The remainder of this section outlines the paper and the results contained herein. The required ideas from the theories of Heegaard splittings and normal surfaces are laid out in Sections 2 and 3. Next, blocks and various associated objects are defined in Section 4. These are used in the proof of the main technical proposition:

Proposition 5.1 If $H \subset\left(M, T_{M}\right)$ is an almost normal Heegaard splitting with sufficiently large genus then $H$ has the disjoint curve property.

The rough idea is that a high genus almost normal surface must decompose as a Haken sum. The exchange annuli of this sum should then give the desired pair of disks and disjoint curve. This rough idea is not quite right, of course, and the proof of the proposition occupies the bulk of the paper. An outline is given in Section 5

Now, not every Heegaard splitting is given as an almost normal surface. However, it is a result of Rubinstein and Stocking [20] that every strongly irreducible splitting may be normalized. (Stated as Theorem 3.7 below.) Thus our main result, Theorem 11.1] is a corollary of Proposition [5.1]

Theorem 11.1 Every splitting in $M$ of sufficiently large genus has the DCP. 
That this result is sharp in general is shown by the Casson-Gordon-Parris examples:

Fact There is a closed, Haken three-manifold containing strongly irreducible splittings of arbitrarily large genus. (See [1] or [19].)

Finally, it would be remiss not to mention that Jaco and Rubinstein [6] have claimed a solution to the generalized Waldhausen conjecture:

Conjecture 1.1 If $M$ is closed, orientable, and atoroidal then $M$ contains only finitely many strongly irreducible splittings, up to isotopy, in each genus.

This, when combined with Theorem 11.1] would give:

Conjecture 12.1 In any closed, orientable three-manifold there are only finitely many full Heegaard splittings, up to isotopy.

The paper ends in Section 12 by explaining this and other, related, open questions.

I thank Kevin Hartshorn and David Bachman for many important conversations in which the main question of the paper was formulated. I also thank Yoav Moriah for his valuable comments on an early version of this paper. I owe a further debt of gratitude to my thesis advisor, Andrew Casson.

\section{$2 \quad$ Heegaard splittings}

This section recalls standard notation and reviews several notions from the theory of Heegaard splittings. (For an excellent survey of the subject, see [18.) The slightly nonstandard notions of disjoint and joined essential surfaces are given as well as the concept of a full splitting.

In this paper, $M$ denotes a closed, orientable three-manifold. Typically $N$ will denote a compact, orientable three-manifold with possibly non-empty boundary. Recall that a three-manifold is reducible if it contains an embedded two-sphere which does not bound an embedded three-ball in $M$.

A handlebody is a compact three-manifold which is homeomorphic to a closed regular neighborhood of a finite, polygonal, connected graph embedded in $\mathbb{R}^{3}$. A Heegaard splitting (or simply a splitting), $H \subset M$, is a embedded, orientable, 
separating, closed surface such that the closure of each component of $M \backslash H$ is a handlebody. These are typically denoted $V$ and $W$. The genus of the splitting is the genus of $H, g(H)=1-\chi(V)$.

Suppose $A \subset V$ is a connected and properly embedded surface. Let $\widetilde{A}$ be the frontier of a closed regular neighborhood of $A$, taken in $V$. Then the surface $\widetilde{A}$ is the double of $A$.

Definition If $D$ is a disk properly embedded in a handlebody $V$ then $D$ is essential if $\partial D$ is not null-homotopic in $\partial V$. If $A$ is an annulus properly embedded in $V$ then $A$ is essential if $A$ is incompressible in $V$ and not boundary-parallel ( rel $\partial A$ ) to an annulus embedded in $\partial V$. A properly embedded Möbius band $A \subset V$ is essential exactly when $\widetilde{A}$ is. (Recall that a surface $F$ properly embedded in a compact manifold $N$ is incompressible is for every embedded disk $(D, \partial D) \subset(N, F)$ with $D \cap F=\partial F$ there is an embedded disk $(E, \partial E) \subset(F, \partial D)$. Also, $F \subset N$ is boundary-parallel if there is a subsurface $G \subset \partial N$ with $\partial F=\partial G, F \cup G$ bounds a submanifold of $N$ homeomorphic to the product $F \times I$, and where $F \times\{1\}=F,(F \times\{0\}) \cup(\partial F \times I)=G$.)

Remark 2.1 This nonstandard definition of "essential" is forced on us by the fact that handlebodies do not contain annuli which are simultaneously incompressible and boundary-incompressible. (Recall that a surface $F$, properly embedded in a compact manifold $N$, is boundary-incompressible if for every disk $(D, \alpha, \beta) \subset(N, F, \partial N)$ such that $\partial D=\alpha \cup \beta, \alpha \cap \beta$ equals two points, $D \cap F=\alpha$, and $D \cap \partial N=\beta$ then there is a disk $\left(E, \alpha, \beta^{\prime}\right) \subset(F, \alpha, \partial F)$ with $\partial E=\alpha \cup \beta^{\prime}$.)

Remark 2.2 Suppose that the genus of the handlebody $V$ is greater than one. Suppose that $A \subset V$ is a properly embedded Möbius band. The irreducibility of $V$ implies that $\widetilde{A}$ is incompressible. Also, $\widetilde{A}$ cannot be boundary-parallel as this would imply that $V$ is a solid torus. Hence $A$ is essential in $V$.

If $J \subset V$ is an essential disk, annulus, or Möbius band then $\partial J \subset \partial V$ is a collection of essential simple closed curves.

Definition Fix a Heegaard splitting $H \subset M$. Suppose $J$ and $K$ are each an essential disk, annulus, or Möbius band in the handlebodies $V$ and $W$, respectively. If $\partial J \subset H$ and $\partial K \subset H$ have a component in common then $H$ admits a joined $J / K$ pair. If $J$ has a boundary component which is disjoint from some boundary component of $K$ then $H$ admits a disjoint $J / K$ pair. 
With this terminology a splitting $H$ is reducible if $H$ admits a joined disk/disk pair. If not reducible, then a splitting is irreducible. A formulation of Haken's Lemma [3] is then:

Lemma 2.3 If $M$ is reducible then every Heegaard splitting $H \subset M$ is reducible.

As a generalization of reducibility Casson and Gordon [2] give the following fruitful notion:

Definition A splitting $H \subset M$ is weakly reducible if $H$ admits a disjoint disk/disk pair. If $H$ is not weakly reducible then it is strongly irreducible.

If $H$ admits a joined disk/annulus pair then, after boundary-compressing the annulus, $H$ admits a disjoint disk/disk pair. Thus $H$ is weakly reducible. If $H$ admits a joined disk/Möbius pair then, as $H$ has genus two or higher, $M$ has $\mathbb{R P}^{3}$ as a connect summand and $H$ is reducible. A somewhat deeper observation is the "no nesting" lemma, due to Scharlemann (see Lemma 2.2 of [17]):

Lemma 2.4 Suppose that $H \subset M$ is a strongly irreducible splitting and $D \subset M$ is an embedded disk, with interior $(D)$ transverse to $H$, and with $\partial D \subset H$. Then $\partial D$ bounds a disk embedded in $V$ or $W$.

Thompson 21] generalizes weak reducibility as follows:

Definition A Heegaard splitting $H \subset M$ has the disjoint curve property if there is an essential simple closed curve $\gamma \subset H$ and a pair of essential disks $D \subset V, E \subset W$ where

$$
D \cap \gamma=\gamma \cap E=\emptyset .
$$

A splitting is full if $H$ does not admit such a triple.

Remark 2.5 This notation is prompted by the fact that if $H$ is full then whenever $D \subset V$ and $E \subset W$ are essential disks the curves $\partial D$ and $\partial E$ fill the splitting surface. That is, $H \backslash(\partial D \cup \partial E)$ is a collection of disks.

Remark 2.6 If $H$ admits a disjoint disk/annulus pair, a joined annulus/annulus pair, or a joined annulus/Möbius band pair then $H$ has the disjoint curve property (DCP). This is shown by boundary-compressing the annuli (or the double of the Möbius band) to obtain the required disks. The required curve $\gamma$ is the appropriate boundary component of the essential annulus. (Joined Möbius pairs and disjoint disk/Möbius pairs do not arise in our proof.) 
Recall that if $M$ is closed, orientable, irreducible, and contains an embedded $\pi_{1}$-injective torus then $M$ is toroidal. Work of Kobayashi [9] shows that if $H$ is a strongly irreducible splitting of a toroidal manifold then $H$ admits a joined annulus/annulus pair. Equipped with this Thompson [21] obtains:

Lemma 2.7 If $M$ is toroidal then every Heegaard splitting $H \subset M$ has the disjoint curve property.

See Figure 1 for a diagram of the relations between the various properties of Heegaard splittings.

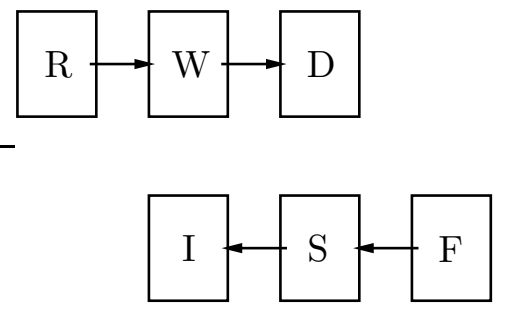

Figure 1: Reducibility implies weakly reducibility which in turn implies the DCP. Also fullness implies strong irreducibility which in turn implies irreducibility. It is an amusing exercise to find a Heegaard splitting for every column.

We end this section with an observation of Hempel's [5] which relies heavily on the classification of Heegaard splittings of Seifert fibred spaces [14:

Lemma 2.8 If $M$ is a Seifert fibred space then every Heegaard splitting $H \subset$ $M$ has the disjoint curve property.

\section{$3 \quad$ Normal surface theory}

This section presents the necessary tools from normal surface theory. For a more complete treatment consult [7, 4], or [8]. See [16] for an introduction to almost normal surfaces.

Fix a triangulation, $T_{N}$, of $N$, a compact orientable three-manifold. Denote the $i$-skeleton of $T_{N}$ by $T_{N}^{i}$. Suppose $F \subset N$ is an properly embedded surface.

Definition The weight of $F, w(F)$, is the number of intersections between $F$ and the one-skeleton of $T_{N}$. The boundary-weight, $w(\partial F)$, is the number of intersections between $\partial F$ and the one-skeleton of $\partial N$. 
The surface $F$ is normal if $F$ is transverse to the skeleta of $T_{N}$ and intersects each tetrahedron in a collection of normal disks. See Figure 2 for pictures of the two kinds of normal disks; the normal triangle and the normal quadrilateral. The value of normal surfaces is amply demonstrated by the following "normalization" lemma, essentially due to Haken [3]:
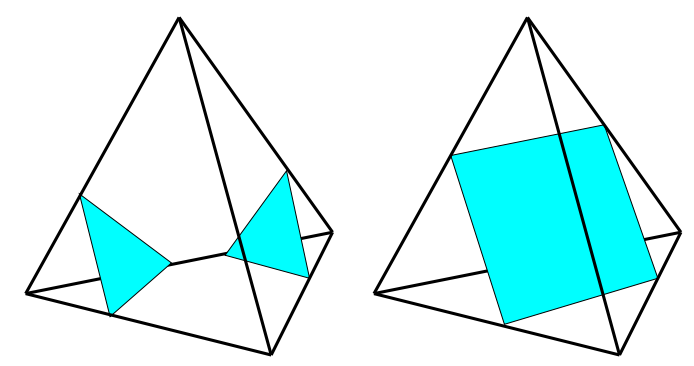

Figure 2: Normal disks

Lemma 3.1 Suppose that $\left(N, T_{N}\right)$ is compact, orientable, irreducible, and boundary-irreducible. If $F \subset N$ is properly embedded, incompressible, and boundary-incompressible then $F$ is properly isotopic to a normal surface $F^{\prime}$ with $w\left(F^{\prime}\right) \leq w(F)$ and with $w\left(\partial F^{\prime}\right) \leq w(\partial F)$. Furthermore, if $F \cap T_{N}^{2}$ contains an arc with both endpoints in a single edge of some face, then also have $w\left(F^{\prime}\right)<w(F)$.

(Recall that a three-manifold $N$ is boundary-irreducible if $\partial N$ is incompressible in $N$.) To illustrate the final sentence of the lemma see Figure 3. Note that the "normalization" lemma holds even for one-sided $F$.

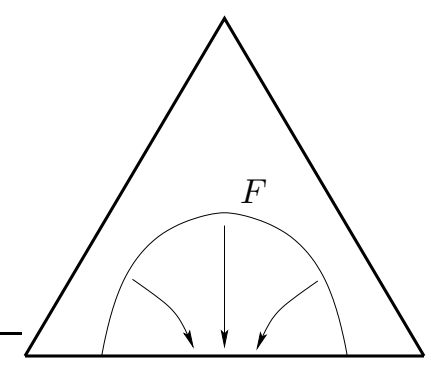

Figure 3: A weight-reducing isotopy

A normal isotopy of $N$ fixes every simplex of $T_{N}$ setwise. Two normal surfaces are equivalent if there is a normal isotopy taking one to the other. Two normal 
surfaces, $J, K \subset N$, are compatible if in each tetrahedron of $T_{N}^{3}$ the surfaces $J$ and $K$ have the same types of quad (or one or both have no quads). In this situation form the Haken sum $F=J+K$ as follows:

Normally isotope $J$ to make $J$ transverse to $K$, to make $\Gamma=J \cap K$ transverse to the skeleta of $T_{N}$, and to minimize the number of components of $\Gamma$. The curves $\gamma \subset \Gamma$ are the exchange curves. For every such curve $\gamma$ let $R(\gamma)$ be the closure (taken in $N$ ) of $\eta_{N}(\gamma)$, an open regular neighborhood of $\gamma$. Since $N$ is assumed orientable $R(\gamma)$ is a solid torus or a one-handle, with $\gamma$ as its core curve.

Now, $\operatorname{fr}_{N}(R(\gamma)) \backslash(J \cup K)$, the frontier of $R(\gamma)$ in $N$ minus the two surfaces, is a union of annuli or rectangles because $N$ is orientable. Taking closures, divide these into two sets, the regular bands $A_{r}(\gamma)$ and the irregular bands $A_{i}(\gamma)$, as indicated by Figure 4. (The fact that $J$ and $K$ are compatible proves that each band receives the same label from every face of $T_{N}^{2}$.) Finally, as in Figure 5 , form the Haken sum:

$$
F=\left((J \cup K) \backslash \bigcup_{\Gamma}\left\{\eta_{N}(\gamma)\right\}\right) \cup \bigcup_{\Gamma}\left\{A_{r}(\gamma)\right\} .
$$

Definition Each connected component of $(J \cup K) \backslash \bigcup_{\Gamma}\left\{\eta_{N}(\gamma)\right\}$ is a patch of the sum $J+K$ while each regular band $A_{r}(\gamma)$ is a seam.

Note that $F$ is again a normal surface which, up to normal isotopy, does not depend on the choices permitted by the above construction. Also, the weight, boundary-weight, and Euler characteristic of $F$ are additive with respect to Haken sum.

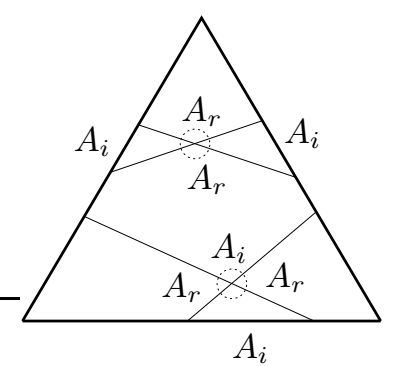

Figure 4: Regular and irregular annuli

Remark 3.2 If $A$ is a seam of $F=J+K$ then $A$ is not a Möbius band. Equivalently, if $A \cap \partial N=\emptyset$ and $\alpha$ is the core curve of $A$ then $\alpha$ preserves 
orientation in $F$. This is trivial when, as with us, $N$ is orientable. (However it also holds for $N$ non-orientable.)

A normal surface is fundamental if it admits no such decomposition. A crucial finiteness result due to Haken (and recorded in [8] ) is:

Lemma 3.3 For a fixed compact, triangulated three-manifold $\left(N^{3}, T_{N}\right)$ there are only finitely many fundamental normal surfaces, up to normal isotopy.

A bit of linear algebra and a few estimates give a more quantitative result (see Hass et. al. 4]):

Lemma 3.4 If $F \subset\left(N, T_{N}\right)$ is fundamental then $w(F) \leq \exp \left(14\left|T_{N}^{3}\right|\right)$. Also, $\chi(F) \geq-\exp \left(14\left|T_{N}^{3}\right|\right)$.

Here $\exp (x)=2^{x}$ is the exponential function and $\left|T_{N}^{3}\right|$ is the number of tetrahedra in the triangulation $T_{N}$. Abusing notation $|\cdot|$, below, also denotes the number of components of a topological space.

Each "cut-and-paste" operation involved in the Haken sum $F=J+K$ may be recorded by an embedded exchange band $C(\gamma)$. The band $C(\gamma)$ is defined as follows: Choose a homeomorphism between $R(\gamma)$, the neighborhood of the intersection curve $\gamma$, and a square-bundle over $\gamma$. This square-bundle has in each fibre (i.e. each square) $J \cup K$ as the two diagonals, $A_{r}(\gamma)$ as one set of opposing sides, and $A_{i}(\gamma)$ as the other set of sides. The exchange band $C(\gamma)$ is the surface which intersects each square in the line segment connecting midpoints of the $A_{r}(\gamma)$ sides. See Figure 5 .

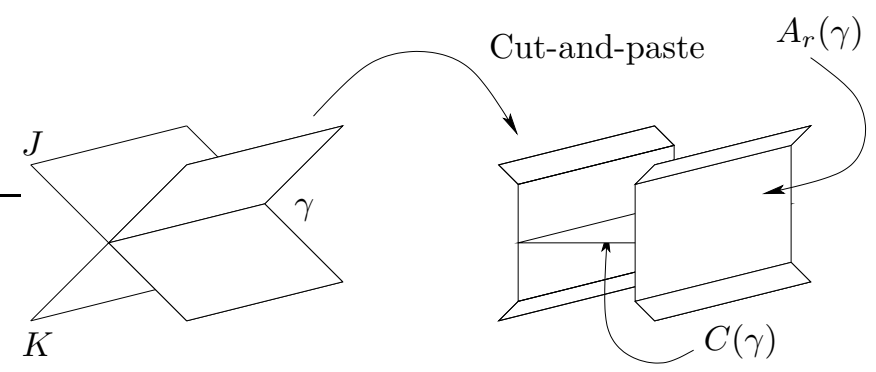

Figure 5: The exchange band for $\gamma$

Each $C(\gamma)$ is an annulus (Möbius band) when $\gamma$ is closed and orientationpreserving (reversing) in $J$ and $K$. The band $C(\gamma)$ is a rectangle when $\gamma$ is 
not closed. Every seam $A_{r}(\gamma)$ is a closed regular neighborhood (in $F$ ) of a boundary component of some $C(\gamma)$.

A sum $F=J+K$ is reduced if the normal surface $F$ cannot be realized as a sum $J^{\prime}+K^{\prime}$ where $J^{\prime}$ and $K^{\prime}$ are again normal, properly isotopic to $J$ and $K$ respectively, with $\left|J^{\prime} \cap K^{\prime}\right|<|J \cap K|$. Note that the isotopy between $J$ and $J^{\prime}$ ( $K$ and $K^{\prime}$ ) need not be normal. The "no disk patches" lemma is a version of a key technical result proved by Jaco and Oertel (Lemma 2.1 of [7] ):

Lemma 3.5 Suppose that $\left(N, T_{N}\right)$ is a triangulated, compact, orientable, irreducible, boundary-irreducible three-manifold. Suppose that $F \subset\left(N, T_{N}\right)$ is a properly embedded, incompressible, boundary-incompressible normal surface which minimizes the lexicographic complexity $(w(\partial F), w(F))$ in its isotopy class. Suppose $F=J+K$ is a reduced sum. Then no patch of $J+K$ is a disk.

Notice that $F$ need not be two-sided. Also, this paper only uses the lemma in the situation $\partial K=\emptyset$.

Remark 3.6 Also contained in [7] is the following observation. Suppose $F=$ $J+K$ is given, as above. Then no exchange annulus $A$ of $J+K$ will be parallel into $F$. For suppose that $(A, \partial A) \subset(N, F)$ was parallel through a solid torus, in the complement of $F$, to an annulus $(C, \partial C) \subset(F, \partial A)$. Then let $F^{\prime \prime}$ be a slight push-off of $(F \backslash C) \cup A$ (see Figure 16) and note that either $w\left(F^{\prime \prime}\right)<w(F)$ or the final line of Lemma 3.1 applies to $F^{\prime \prime}$. In either case a contradiction is obtained.

A surface $H$, embedded in a closed manifold $\left(M, T_{M}\right)$, is almost normal if $H$ is transverse to the skeleta of $T_{M}$ and intersects each tetrahedron but one in a collection of normal disks. In the remaining tetrahedron $H$ yields a collection of normal disks and at most one almost normal piece. Two of the five kinds of almost normal pieces are shown in Figure [6. The first is the almost normal octagon while the second is one of the almost normal annuli. The other possibilities are an almost normal annulus between triangles of the same type, between a pair of quads, or between a triangle and a quad.

The following theorem (see the papers of Rubinstein [16] and Stocking [20]) is another important normalization result:

Theorem 3.7 If $H \subset\left(M, T_{M}\right)$ is a strongly irreducible Heegaard splitting then $H$ is isotopic to an almost normal surface. 

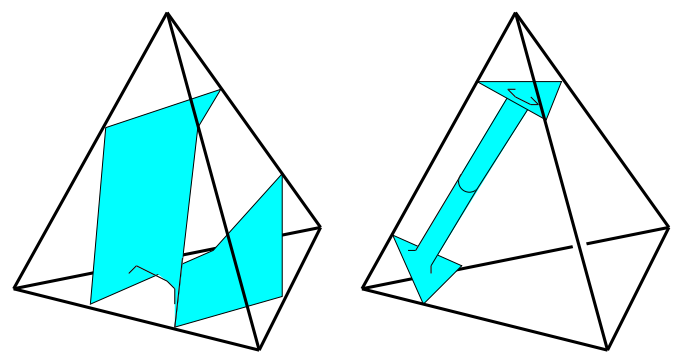

Figure 6: Almost normal pieces

\section{Blocked and shrunken submanifolds}

This section introduces blocks and deals with submanifolds (of a triangulated three-manifold) which are naturally contained in unions of blocks.

Suppose that $\tau$ is a regular Euclidean tetrahedron. Let $S \subset \tau$ be a disjoint collection of normal disks together with at most one almost normal piece. ( $S=$ $\emptyset$ is allowed.)

Definition A block, $B$, is the closure of a connected component of $\tau \backslash S$. Suppose $B$ is adjacent to exactly two normal disks, $D$ and $E$. Suppose also that $D$ is normally isotopic in $\tau$ to $E$. Then $B$ is a product block. All other blocks are called core blocks.

See Figure 7 for pictures of the two product blocks and six of the many possible core blocks. Note that a few of the faces are shaded - these are the faces which lie in the surface $S$. Such faces form the horizontal boundary of the block while the faces $B \cap \tau^{2}$, in the two-skeleton, are the vertical boundary of $B$. Define a vertical rectangle of a block $B$ to be any face of the vertical boundary which is disjoint from the zero-skeleton of $T_{M}$ and which has four sides. For example, all vertical faces of a product block are rectangles.

Let $\left(M, T_{M}\right)$ be a closed, orientable, triangulated three-manifold.

Definition A three-dimensional submanifold $X \subset\left(M, T_{M}\right)$ is blocked if $\partial X$ is an almost normal surface and $X$ is a union of blocks.

As a specific example, if $H \subset\left(M, T_{M}\right)$ is connected, separating, normal surface then the closure of a component of $M \backslash H$ is a blocked submanifold. 

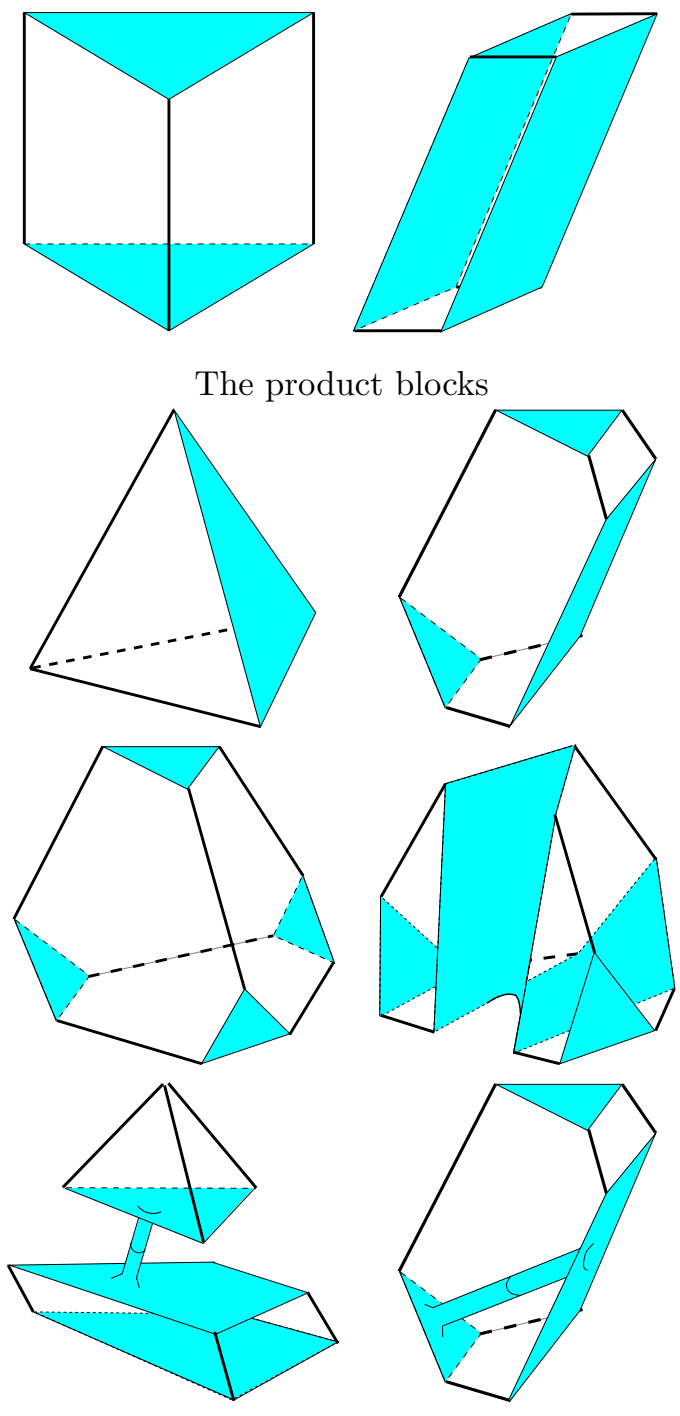

A few core blocks

Figure 7: Some of the possible blocks (not all to scale)

Remark 4.1 Note that a blocked submanifold contains at most $6 t+1$ core blocks, where $t=\left|T_{M}^{3}\right|$ is the number of tetrahedra in $T_{M}$. To see this, note that a tetrahedron contains at most five parallel families of normal disks. Thus any tetrahedron not meeting an almost normal piece contributes at most six core blocks. The almost normal piece (if it exists) gives the final core block. 
Suppose that $X \subset\left(M, T_{M}\right)$ is blocked. Suppose $Y \subset X$ is a subset of $X$. Define the horizontal boundary of $Y$ to be $\partial_{h} Y=Y \cap \partial X$. Also define the vertical boundary of $Y$ to be $\partial_{v} Y=\operatorname{fr}_{X}(Y)$, the set of points in $Y$ which do not have an open neighborhood (relative to $X$ ) in $Y$.

Recall that $\eta_{X}(\cdot)$ denotes a open regular neighborhood taken inside of $X$.

Definition Let $X \subset\left(M, T_{M}\right)$ be blocked. A three-dimensional submanifold $Y \subset X$ is shrunken if there is a union of blocks $\widehat{Y} \subset X$, with

$$
Y=\widehat{Y} \backslash \eta_{X}\left(\partial_{v} \widehat{Y}\right)
$$

Thus the shrunken $Y$ is obtained by removing a small neighborhood of $\partial_{v} \widehat{Y}$ from $\widehat{Y}$. (That is, by shrinking $\widehat{Y}$.) Note that $Y$ uniquely determines $\widehat{Y}$ as well as the reverse.

Definition If $Y$ is any submanifold of a blocked $X \subset\left(M, T_{M}\right)$ then define the complexity of $Y$ :

$$
c(Y)=\chi(Y)+\left|\partial_{v} Y\right|
$$

This is an exact parallel of the complexity of a compact surface $G, c(G)=$ $\chi(G)+|\partial G|$. If $Y$ is an $I$-bundle then $c(Y)$ agrees with the complexity of the base surface.

If $Y$ is connected and $c(Y) \leq 0$ then $Y$ is large. Again, this parallels the notation for surfaces: A connected surface $G$ is large if $G$ has nonpositive complexity. Equivalently, $G$ is large if $G$ contains an orientation-preserving, nonseparating, simple closed curve.

We end this section with a discussion of how to obtain new shrunken submanifolds from old. Let $Y$ be a shrunken submanifold of the blocked $X$. Let $B \subset X$ be a block which non-trivially intersects the interior of $Y$. Then $Y \backslash \eta_{X}(B)$ is again a shrunken submanifold inside of $X$. The following lemma records how the complexity of $Y \backslash \eta_{X}(B)$ differs from that of $Y$ :

Lemma 4.2 Given a blocked $X \subset\left(M, T_{M}\right)$, a shrunken submanifold $Y \subset X$, and a block $B$ of $X$ which non-trivially intersects $Y$ then:

$$
c\left(Y \backslash \eta_{X}(B)\right) \leq c(Y)+32 .
$$

Proof First note that the block $B$ contains at most four vertices of $T_{M}^{0}$. Also, $B \cap\left(T_{M}^{1} \backslash T_{M}^{0}\right)$ is the union of at most 12 connected arcs. This is because $B$ 
intersects any single edge of $T_{M}^{2}$ at most twice. Finally, $\partial_{v} B$ contains at most 12 faces. To see this last, note that the dual graph to $B \cap T_{M}^{2}$ has as at most 12 edges, dual to the components of $B \cap\left(T_{M}^{1} \backslash T_{M}^{0}\right)$, and this graph has no vertices of valency zero or one.

To obtain $Y \backslash \eta_{X}(B)$ remove neighborhoods of certain skeleta of $B$ from $Y$. To be precise, for every vertex in $B \cap T_{M}^{0} \cap Y$ remove a small open neighborhood of the vertex from $Y$. Let $Y^{\prime}$ be the compact submanifold obtained. Each vertex removed increases the Euler characteristic by one and contributes one additional vertical boundary component. Thus,

$$
c\left(Y^{\prime}\right) \leq c(Y)+8 \text {. }
$$

Remove from $Y^{\prime}$ a regular neighborhood of $\left(B \cap Y^{\prime}\right) \cap T_{M}^{1}$, giving the submanifold $Y^{\prime \prime}$. Note that $c\left(Y^{\prime \prime}\right) \leq c\left(Y^{\prime}\right)$ as any increase in $\left|\partial_{v}\right|$ is exactly compensated by a decrease in Euler characteristic.

Finally remove a regular neighborhood of $\left(B \cap Y^{\prime \prime}\right) \cap T_{M}^{2}$ to obtain $Y^{\prime \prime \prime}$. There are at most 12 faces to remove; each increases Euler characteristic and $\left|\partial_{v}\right|$ by at most one. Thus,

$$
c\left(Y^{\prime \prime \prime}\right) \leq c\left(Y^{\prime \prime}\right)+24 .
$$

Deleting the interior of $B$ yields $Y \backslash \eta_{X}(B)$ and does not increase the complexity. To see this note that the Euler characteristic remains unchanged when $B$ is a solid torus and actually decreases when $B$ is a ball. Also, removing the interior of $B$ discards at least one component of the vertical boundary. Thus $c\left(Y \backslash \eta_{X}(B)\right) \leq c(Y)+32$, as desired.

\section{The main proposition}

Here the main technical proposition is given. As the proof of the proposition is quite long it is broken apart among the sections which follow.

Proposition 5.1 Suppose $\left(M, T_{M}\right)$ is a closed, orientable, triangulated threemanifold. Suppose $H \subset M$ is an almost normal Heegaard splitting with genus $g(H)>\exp \left(2^{16} t^{2}\right)$. Then $H$ has the disjoint curve property.

Here $t=\left|T_{M}^{3}\right|$ is the number of tetrahedra in $T_{M}$ and, again, $\exp (x)=2^{x}$ is the exponential function.

Remark 5.2 The estimate on genus is extremely wasteful. However, our methods cannot be used to give upper bounds better than $\exp \left(c \cdot t^{2}\right)$ where $c$ is a constant. See also the discussion in Section 12 
We now outline the proof of Proposition 5.1, which operates via contradiction.

First assume that an almost normal Heegaard splitting $H$ is given which both has large enough genus and is full. Both hypotheses are used throughout the proof. Cut $M$ along $H$ to obtain a pair of blocked handlebodies, $V$ and $W$. Section [6] and Section 7 produce a shrunken $I$-bundle $Y \subset V$ with $\partial_{h} Y \subset H$. The $I$-bundle $Y$ has $\chi(Y) \leq-\exp \left(2^{15} t^{2}\right)$ while still having a small number of fairly short vertical boundary annuli. Furthermore, it is shown that the vertical and horizontal boundaries of $Y$ are incompressible inside of $V$.

Remark 5.3 Note that, when $Y$ is not a twisted $I$-bundle, the proof becomes quite straight-forward. It is easy to find an essential annulus in $W$ with one boundary component in $\partial_{h} Y$. Simply take this curve and "push it through" $Y$ to obtain the desired joined annulus/annulus pair.

Next, in Section 8, a manifold $N \cong M \backslash \eta_{M}\left(\partial_{v} Y\right)$ is introduced and shown to be irreducible and boundary-irreducible. Also, a section $F$ of the $I$-bundle $Y$ is chosen and shown to be incompressible and boundary-incompressible inside of $N$.

The manifold $N$ is triangulated in Section 9. The derived triangulation $T_{N}$ has at most $2^{9} t^{2}$ tetrahedra where $t=\left|T_{M}^{3}\right|$. This bound follows from the small size of $\partial_{v} Y$. The surface $F$ is then normalized with respect to $T_{N}$ so that $w(\partial F) \leq 2^{6} t^{2}$. This, together with the fact that $\chi(F)=\chi(Y)$, allows a Haken decomposition of $F$ of a pleasant form. Note that $F$ cannot be a fundamental normal surface in $N$, as the Euler characteristic of $F$ is too negative.

Finally, in Section 10, the exchange bands of the decomposition of $F$ are examined. It is one of these which gives rise to an joined annulus/annulus (or Möbius/annulus) pair for the splitting surface $H$. Boundary-compressing each of these essential annuli (or the double of the Möbius band) shows that $H$ had the disjoint curve property, a final contradiction. This will complete the proof of Proposition [5.1].

\section{$6 \quad$ Shelling high genus splittings}

This section deals with one of the main objects of interest: almost normal Heegaard splittings. A pair of blocked handlebodies and a few shrunken submanifolds will be derived from such a splitting. These submanifolds will be large $I$-bundles and their vertical annuli provide our first supply of essential 
annuli. The constructions and notations here introduced will be used heavily throughout the proof of Proposition 5.1 .

Suppose that $\left(M, T_{M}\right)$ is a closed, orientable, triangulated three-manifold and $H$ is an almost normal splitting with genus greater than $\exp \left(2^{16} t^{2}\right)$ as given by Proposition [5.1. Recall that $t=\left|T_{M}^{3}\right|$. Suppose also that $H$ has the smallest weight among all almost normal surfaces isotopic to $H$. Finally suppose, for an eventual contradiction, that $H$ is a full splitting.

First analyze the basic structure of $M$. From Haken's Lemma (Lemma 2.3 above) and the fact that full splittings are irreducible it follows that:

Claim 6.1 The manifold $M$ is irreducible.

From Waldhausen's Theorem [22] further deduce:

Claim 6.2 The manifold $M$ is not homeomorphic to the three-sphere.

Recall that $H$ divides $M$ into two blocked handlebodies, $V$ and $W$. Let $\widehat{V_{1}}$ be the union of all product blocks in $V$. Let $\widehat{W_{2}}$ be the union of all product blocks $B \subset W$ such that $\partial_{h} B \subset \partial_{h} \widehat{V_{1}}$. Let $V_{1}$ and $W_{2}$ be the corresponding shrunken submanifolds inside of $V$ and $W$. As $M$ is orientable, $V_{1}$ and $W_{2}$ are orientable submanifolds. Also, by construction, $\partial_{h} W_{2} \subset \partial_{h} V_{1}$.

Let $Z_{2} \subset W_{2}$ be a connected component which has smaller (more negative) complexity than any other component of $W_{2}$. Amongst the one or two components of $V_{1}$ meeting $\partial_{h} Z_{2}$, let $Y_{1}$ be one with smallest complexity.

We investigate the properties of these $I$-bundles, in several steps.

Claim 6.3 The $I$-bundle $W_{2}$ has $c\left(W_{2}\right) \leq 1-g(H)+20 \cdot 32 t$. Also, $\left|\partial_{v} W_{2}\right|<$ $104 t$.

Proof The $I$-bundle $W_{2}$ is obtained from $W$ by removing all core blocks from $W$ and then removing all product blocks of $W$ which are adjacent to core blocks of $V$, across a horizontal face. There are at most $6 t+1$ of the former by Remark 4.1. It remains to estimate the number of the latter. If the almost normal piece sits inside the tetrahedron $\tau$ then $\tau$ contains at most six families of parallel product blocks. If not, then $\tau$ contains at most five such families. Each family of parallel product blocks contains at most two blocks adjacent to a core block. Thus, the $I$-bundle $W_{2}$ is obtained by removing at most another 
$10 t+2$ product blocks from $W$. Applying Lemma 4.2 the complexity of $W_{2}$ is bounded by

$$
c\left(W_{2}\right) \leq 1-g(H)+20 \cdot 32 t .
$$

To obtain the second inequality of the claim: note that each core block has at most 8 vertical rectangles: those four-sided vertical faces which do not meet the zero-skeleton of $T_{M}$. Product blocks have either 3 or 4 vertical rectangles. Each contributes at most one to the "length" of $\partial_{v} W_{2}$, so it certainly follows that $\left|\partial_{v} W_{2}\right| \leq 8(6 t+1)+4(10 t+2) \leq 104 t$.

Recall that $Z_{2}$ was chosen to be a component of $W_{2}$ with smallest (most negative) complexity.

Remark 6.4 By the above claim the $I$-bundle $W_{2}$ contains at most $104 t$ components. Thus, as complexity is additive over disjoint union, the component $Z_{2}$ has complexity

$$
c\left(Z_{2}\right) \leq \frac{c\left(W_{2}\right)}{104 t} \leq \frac{1-g(H)}{104 t}+8 .
$$

Recall that $Y_{1}$ is a component of $V_{1}$ such that $\partial_{h} Y_{1}$ contains a component of $\partial_{h} Z_{2}$.

Remark 6.5 The complexity of $Y_{1}$ is bounded above by:

$$
c\left(Y_{1}\right) \leq \frac{1}{2} c\left(Z_{2}\right) \leq \frac{1-g(H)}{208 t}+4 .
$$

This follows from Lemma A.1 proved in the appendix, applied to the relevant components of $\partial_{h} Y_{1}$ and $\partial_{h} Z_{2}$. Conclude that $c\left(Y_{1}\right) \leq-\exp \left(2^{15} t^{2}\right)$.

The $I$-bundles $Y_{1}$ and $Z_{2}$ both contain vertical nonseparating annuli, say $A \subset$ $Y_{1}$ and $B \subset Z_{2}$. Applying Lemma B.1 from the appendix find:

Claim 6.6 There are essential annuli $A \subset V, B \subset W$ such that $\partial A$ and one component of $\partial B$ are embedded in $\partial_{h} Y_{1}$.

From this immediately deduce a non-essential but technically useful corollary.

Claim 6.7 The almost normal splitting $H$ does not contain an almost normal annulus. 
Proof Recall that $H$ was assumed, at the beginning of this section, to be the least weight surface among all almost normal surfaces isotopic to $H$. Suppose that the splitting $H$ contains an almost normal annulus. Then there is a disk $D \subset M$ such that $\partial D$ is a core curve for the almost normal annulus, $D$ meets $H$ only along $\partial D$, and the disk $D$ does not meet the two-skeleton $T_{M}^{2}$.

There are two possibilities: either $\partial D$ is inessential in $H$ or it is essential. If inessential then surger $H$ along $D$ to obtain $H^{\prime}$. The surface $H^{\prime}$ is the union of a normal two-sphere $S$ and a normal surface $H^{\prime \prime}$. As $M$ is irreducible (Claim 6.1) the two-sphere $S$ bounds a three-ball $U \subset M$. If the splitting $H$ were contained in $U$ then $M \cong S^{3}$. This is impossible by Claim 6.2. Deduce that there is an isotopy taking $H^{\prime \prime}$ to $H$ which is supported on a regular neighborhood of $U$. But this is another impossibility as $H^{\prime \prime}$ is normal with $w\left(H^{\prime \prime}\right)<w(H)$.

It follows that $\partial D$ is essential in $H$. Note that $\partial D \cap \partial_{h} V_{1}=\emptyset$. By Claim 6.6 $D$ forms one-half of a disjoint disk/annulus pair. Boundary-compressing the given annulus demonstrates that $H$ has the DCP, another contradiction.

This proof exemplifies a theme of this paper - always seek to compare a troublesome disk to a vertical annulus provided by one of the $I$-bundles. As $H$ is full it cannot admit a disjoint disk/annulus pair. Conclude that the vexatious disk does not exist.

\section{Capping off to obtain $Y$}

This section discusses the annuli forming the vertical boundary of $Y_{1}$. Some of these bound two-handles in $V$. These two-handles will be added to $Y_{1}$ to form a bigger $I$-bundle, $Y$.

For every vertical annulus $C_{i} \subset \partial_{v} Y_{1}$, with both components of $\partial C$ inessential in $H$, let $D_{i}$ and $D_{i}^{\prime}$ be the disks in $H$ which the components of $\partial C_{i}$ bound. The two-sphere $C_{i} \cup D_{i} \cup D_{i}^{\prime}$ bounds a ball, $U_{i} \subset V$, as handlebodies are irreducible. Note that $U_{i} \cap Y_{1}=C_{i}$. (If not $Y_{1} \subset U_{i}$ and $\partial_{h} Y_{1}$ would be planar, contradicting the fact that $Y_{1}$ is large.)

Give each ball $U_{i}$ the structure of an $I$-bundle over the disk $\mathbb{D}^{2}$. Arrange matters so that the $I$-bundle structure on $C_{i}$ coming from $Y_{1}$ and $U_{i}$ agree. Let $Y=Y_{1} \cup \bigcup_{i} U_{i}$. Again, $Y$ is a shrunken submanifold of $V, Y$ is a large $I$-bundle, and $\partial_{h} Y_{1} \subset \partial_{h} Y$. We are now equipped to make several observations about the structure of $Y$. 
First of all, $c(Y)=c\left(Y_{1}\right) \leq-\exp \left(2^{15} t^{2}\right)$. Also, $\partial_{v} Y$ is non-empty. (Because surface groups are not free!)

Remark 7.1 Note that $\partial_{h} Y_{1} \subset \partial_{h} Y$. Thus Claim [6.6 applies to $Y$ just as it applies to $Y_{1}$. That is, there are essential annuli $A \subset V, B \subset W$ such that $\partial A$ and one component of $\partial B$ are embedded in $\partial_{h} Y$.

Definition The corners of the $I$-bundle $Y$ are the components of $\partial\left(\partial_{v} Y\right)$.

Claim 7.2 No corner of $Y$ bounds an embedded disk in $M$ which is transverse to $H$. (So, in particular, the corners of $Y$ are essential in $H$.)

Proof Fix attention on $C$, a component of $\partial_{v} Y$. Let $\partial C=\gamma \cup \gamma^{\prime}$. Suppose, for a contradiction, that $\gamma$ bounds a disk in $M$ which is transverse to $H$. By taking the union of this disk with $C$ and performing a small isotopy $\gamma^{\prime}$ also bounds a disk in $M$, transverse to $H$.

By the construction of $Y$ one component of $\partial C$ is essential in $H$. Apply "no nesting" (Lemma 2.4) to this essential boundary component. It follows that $Y$ has a corner which is essential in $H$ and bounds a disk in $V$ or $W$. By Remark 7.1 there is a disjoint disk/annulus pair and so $H$ has the DCP. This is a contradiction.

It immediately follows that:

Claim 7.3 The components of $\partial_{v} Y$ are incompressible in $V$.

Also:

Claim 7.4 The components of $\partial_{h} Y$ are incompressible in $V$.

Proof Let $D \subset V$ be a compressing disk for $\partial_{h} Y$ which minimizes $\left|D \cap \partial_{v} Y\right|$. Then $D$ does not meet $\partial_{v} Y$ by Claim 7.3 . Thus $D$ lies inside of $Y$. But this is impossible, as the horizontal boundary of an $I$-bundle is always incompressible inside the $I$-bundle. 


\section{Focusing on the middle surface}

This section discusses the geometric structure of a section $F \subset Y$. This surface is properly embedded in the submanifold $N=\overline{M \backslash R}$, as discussed below.

Let $Y \subset V$ be the $I$-bundle produced above, in Section 7 . Let $R$ be the following union of closed solid tori:

$$
R=\overline{\eta_{\overline{V \backslash Y}}\left(\partial_{v} Y\right)}
$$

Then $R \cap Y=\partial_{v} Y$. That is, if $C$ is a component of $\partial_{v} Y$ and $R^{\prime}$ is the component of $R$ meeting $C$ then $R^{\prime}$ is a collar for $C$ inside of $\overline{V \backslash Y}$. See Figure 8

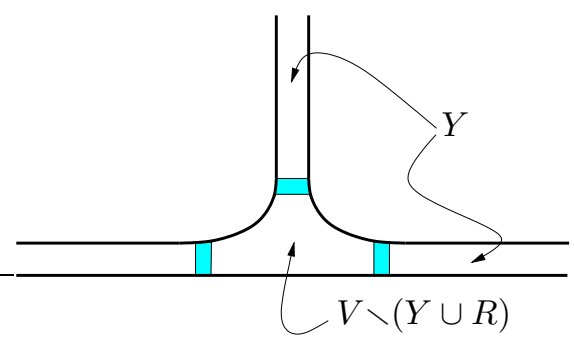

Figure 8: A cross-section of $V$. The union of tori, $R$, is shaded.

Let $F \subset Y$ be a properly embedded surface, transverse to the fibres of $Y$, meeting each fibre exactly once, and with $F \cap \partial_{h} Y=\emptyset$. See Figure 9, Let $\pi: Y \rightarrow F$ be the natural bundle map: $\pi^{-1}(x)$ is the $I$-fibre of $Y$ meeting the point $x \in F$. The surface $F$ is a section of the $I$-bundle $Y$.

It follows that the complexity of $F$ equals that of $Y$. The Euler characteristic of $F$ is less than $c(F)=c(Y) \leq-\exp \left(2^{15} t^{2}\right)$ because $\partial F$ is nonempty. Note that $F$ is properly embedded in the three-manifold $N=\overline{M \backslash R}$. When discussing $Y$ inside of $N$ we will regard it as a closed regular neighborhood of $F$. The rest of this section studies the pair $F \subset N$.

Claim 8.1 The surface $F$ is incompressible in $N$.

Before proving this, observe that a stronger statement would be that $\partial_{h} Y$ is incompressible in $W$. However, this does not seem to necessarily hold when $Y$ is a twisted $I$-bundle. 


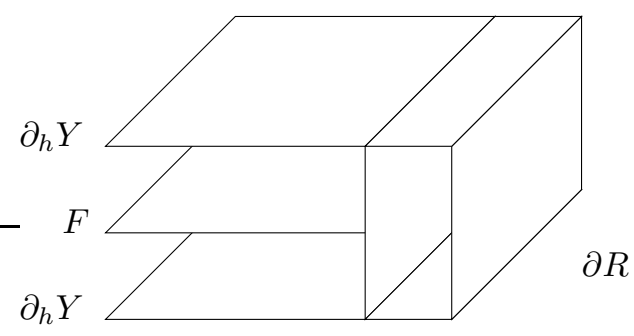

Figure 9: Side-view of $F \subset Y$

Proof of Claim 8.1 Suppose that $F$ is compressible, via a disk $D \subset N$. Let $\alpha=\partial D$ and set $A=\pi^{-1}(\alpha)$. Note that $A$ is an annulus, not a Möbius band. We may assume, after a small isotopy of $D$ rel $\alpha$, that $D \cap Y=\eta_{D}(\alpha) \subset A$.

Let $D^{\prime}=D \backslash \operatorname{interior}(Y)$. Set $\alpha^{\prime}=\partial D^{\prime} \subset \partial_{h} Y$. The curve $\alpha^{\prime}$ is essential in $H$. (If $\alpha^{\prime}$ bounds a disk in $\partial_{h} Y$ then $\alpha$ is null-homotopic in $F$, a contradiction. If $\alpha^{\prime}$ bounds a disk in $H$ meeting $\partial\left(\partial_{h} Y\right)$ then have a contradiction to Claim[7.2)

Lemma 2.4 replaces $D^{\prime}$ by a disk $D^{\prime \prime}$ with $\partial D^{\prime \prime}=\partial D^{\prime}=\alpha^{\prime}$ and with the interior of $D^{\prime \prime}$ disjoint from the splitting $H$. Note that $D^{\prime \prime}$ cannot lie inside of $V$ by Claim [7.4. It follows that $D^{\prime \prime}$ lies in $W$.

Now choose $\beta \subset F \backslash \alpha$ a simple closed curve which is nonseparating and orientation-preserving in $F$. (Such a curve exists as $c(F)$ is very negative.) By Lemma B.1 the annulus $B=\pi^{-1}(\beta)$ is essential in $V$. Thus $D^{\prime \prime} / B$ forms a disjoint disk/annulus pair and $H$ has the DCP. This is a contradiction.

Claim 8.2 The surface $F$ is boundary-incompressible in $N$.

Proof Suppose that $F$ is boundary-compressible, via a disk $D \subset N$. Choose $D$ to minimize the quantity $|\operatorname{interior}(D) \cap H|$. Let $\alpha=(\partial D) \cap F$. We may assume, after a small proper isotopy of $D$ rel $\alpha$ which leaves $|\operatorname{interior}(D) \cap H|$ unchanged, that $D \cap Y=\eta_{D}(\alpha) \subset \pi^{-1}(\alpha)$. Let $D^{\prime}=D \backslash \operatorname{interior}(Y)$. Set $\alpha^{\prime}=D^{\prime} \cap \partial_{h} Y$.

Note that $D^{\prime} \cap H$ contains no simple closed curves embedded in interior $\left(D^{\prime}\right)$. For suppose to the contrary that $\delta$ is such an innermost such curve and $\delta$ bounds a subdisk $E \subset D^{\prime}$. Now $\delta$ must be essential in $H$. (If not $\delta$ bounds a disk disk $E^{\prime} \subset H$. As $\partial_{h} Y$ is nonplanar $E^{\prime} \cap \partial_{h} Y=\emptyset$. So use an innermost disk of $E^{\prime}$ to surger $D$, reducing |interior $(D) \cap H \mid$. This is a contradiction.) Thus $E$ is an essential disk in $V$ or $W$ which is disjoint from $\partial_{h} Y$. By Remark [7.1] 
the splitting $H$ admits a disjoint disk/annulus pair, implying that $H$ has the DCP, a contradiction. So, as desired, $D^{\prime} \cap H$ contains no simple closed curves embedded in interior $\left(D^{\prime}\right)$.

Consider the components of $D^{\prime} \backslash H$. The closure of one of these contains $\alpha^{\prime}$. Denote the closure of that component by $D^{\prime \prime}$. See Figure 10.

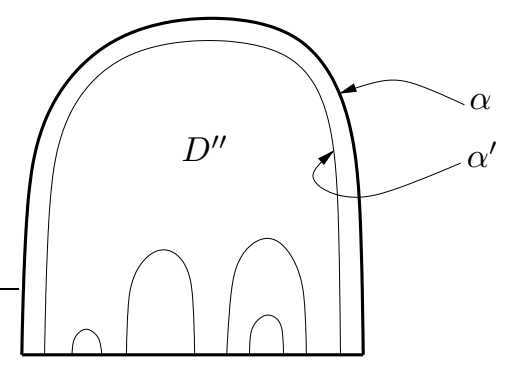

Figure 10: The disk $D$, showing $D \cap H$ and $D^{\prime \prime}$

Note that $D^{\prime \prime}$ is a disk embedded in $W$. Let $\beta \subset F$ be a non-separating, orientation-preserving simple closed curve which is disjoint from $\alpha$. (Such a curve exists as $c(F)$ is very negative.) Set $B=\pi^{-1}(\beta)$; the annulus which is the union of all fibres of $Y$ meeting $\beta$. By Lemma B.1 the annulus $B$ is essential in $V$.

If $D^{\prime \prime}$ were essential then $D^{\prime \prime}$ and $B$ would form a disjoint disk/annulus pair, a contradiction. Thus $\partial D^{\prime \prime}$ bounds a disk $E \subset H$ with $\partial E \cap Y=\alpha^{\prime}$. As $\partial\left(\partial_{v} Y\right) \cap E$ contains no simple closed curves (Claim [7.2), it follows that $\alpha^{\prime}$ is boundary-parallel in $\partial_{h} Y$. So $\alpha$ is homotopic (rel boundary) into $\partial F$. This implies that the original disk $D$ was not a boundary-compression after all.

Before examining the three-manifold $N$ further we will need the following:

Claim 8.3 The $I$-bundle $Y \subset M$ is not contained inside of a three-ball in $M$.

Proof Suppose that $Y$ is contained inside of a three-ball. Pick a three-ball $U \subset M$ such that $U$ contains $Y$ and minimizes $|S \cap H|$, where $S=\partial U$. If $|S \cap H|=0$ then $H$ is contained inside of a ball in $M$. Since handlebodies are irreducible this implies that $M$ is the three-sphere, contradicting Claim 6.2

Thus $S \cap H$ is nonempty. Let $\delta \subset(S \cap H)$ be innermost in $S$. Let $D \subset S$ be an innermost disk which $\delta$ bounds. If $\delta$ is essential in $H$ then, by Remark 7.1 . $D$ is half of a disjoint disk/annulus pair and $H$ has the DCP, a contradiction. 
Thus $\delta$ bounds a disk in $H$. Let $E \subset H$ be an innermost disk of $S \cap H$. Set $\epsilon=\partial E$. The disk $E$ is disjoint from $Y$, as $\partial_{h} Y$ is nonplanar. Let $E^{\prime}$ and $E^{\prime \prime}$ be slight push-offs of $E$. Perform disk surgery on $S$ along $E$ to obtain $S^{\prime} \amalg S^{\prime \prime}=\left(S \backslash \eta_{S}(\epsilon)\right) \cup E^{\prime} \cup E^{\prime \prime}$, a disjoint union of two-spheres. Note that both $\left|S^{\prime} \cap H\right|$ and $\left|S^{\prime \prime} \cap H\right|$ are less than $|S \cap H|$. Also, as $M$ is irreducible, both $S$ and $S^{\prime}$ bound three-balls.

If $E \subset U$ then both $S^{\prime}$ and $S^{\prime \prime}$ bound balls inside of $U$ and one of these contains $Y$. This however contradicts the minimality of $|S \cap H|$. Suppose instead that $E \cap U=\epsilon=\partial E$. If one of $S^{\prime}$ or $S^{\prime \prime}$ bounds a ball containing $U$ then that one of $S^{\prime}$ or $S^{\prime \prime}$ bounds a ball containing $Y$, a contradiction to minimality. It follows that both bound balls disjoint from $U$. Thus $M$ is homeomorphic to the three-sphere. This last contradicts Claim 6.2.

From this, deduce:

Claim 8.4 The three-manifold $N$ is irreducible.

Proof Suppose not. Let $S \subset N$ be a reducing sphere for $N$ which minimizes the quantity $|S \cap F|$.

Suppose that $|S \cap F|=0$. After a small isotopy of $Y$ we may assume that $S \cap Y=\emptyset$, because $Y$ is a regular neighborhood of $F$ in $N$. Now, $S$ bounds a three-ball $U \subset M$, as $M$ is irreducible (Claim 6.1). By Claim 8.3 the $I$-bundle $Y$ does not meet $U$. As $R$ is a small collar of $\partial_{v} Y$ deduce that $U$ is disjoint from the union of solid tori $R$. Thus $U$ embeds in $N=\overline{M \backslash R}$ and $S$ was not a reducing sphere, a contradiction.

Suppose instead that $|S \cap F|>0$. Let $\delta \subset(S \cap F)$ be innermost in $S$, bounding the disk $D \subset S$. As $F$ is incompressible (Claim 8.1) the curve $\delta$ also bounds a disk in $F$. Let $E \subset F$ be an innermost subdisk with boundary $\partial E=\epsilon \subset S \cap F$. As in the proof of Claim 8.3 above, surger $S$ along the disk $E$ to obtain twospheres $S^{\prime}$ and $S^{\prime \prime}$.

If neither of these is a reducing sphere then a standard argument shows that $S$ was also not a reducing sphere, a contradiction. But $S^{\prime}$, say, being a reducing sphere contradicts the minimality assumption.

Finally, deduce that $N$ is boundary-irreducible, or what amounts to the same thing:

Claim 8.5 The boundary of $N$ is incompressible inside of $N$. 
Proof Suppose that $\partial N$ compresses. Let $D$ be a compressing disk for $\partial N$ which minimizes $|D \cap F|$. Note that $D \cap F$ contains no simple closed curves. (If such exists then by the incompressibility of $F$ there is a disk $E \subset F$ with $E \cap D=\partial E$. Surger $D$ along $E$ to reduce $|D \cap F|$.)

Suppose first that $|D \cap F|=0$. Properly isotope $D$ in $N$ to a disk $D^{\prime}$ so that $\partial D^{\prime}$ lies inside some component of $R \cap H$. That is, $\partial D^{\prime}$ lies in $H$ and is parallel to a component of $\partial\left(\partial_{h} Y\right)$. This contradicts Claim 7.2 .

Suppose that $|D \cap F|>0$. Let $D^{\prime \prime} \subset D$ be an outermost bigon with respect to the arcs $D \cap F$. As $F$ is boundary-incompressible the arc $D^{\prime \prime} \cap F$ cuts $F$ into two pieces, one of which is a bigon, $E$. An outermost bigon $E^{\prime} \subset E$ gives a $\partial$-surgery for $D$. Performing this surgery will produce at least one compressing disk for $\partial N$ which meets $F$ in fewer arcs, a contradiction.

\section{Normalizing $F$}

This section finds a triangulation for $N$ and shows that $F$ may be normalized inside of $N$. This normal surface will then decompose as a well-behaved Haken sum. As $F$ is not necessarily two-sided, it is not immediately obvious how to replace the technicalities of triangulations and normal surface theory with a neater proof using branched surfaces. However, I would be happy to be corrected on this point.

Claim 9.1 The manifold $N$ may be triangulated with fewer than $2^{9} t^{2}$ tetrahedra so that the surface $F$ has boundary-weight less than $2^{6} t^{2}$.

Here $t=\left|T_{M}^{3}\right|$ is the number of tetrahedra in the given triangulation of $M$. The proof operates by constructing a sufficiently simple triangulation, $T_{N}$, of $N$. This construction is complicated while also being somewhat uninteresting. I would advise the reader to take the above claim on faith in his or her first reading of this section and instead skip directly to Section 9.5.

\subsection{Constructing the Graph $\Theta_{F}$}

Let $\widehat{Y}$ be the union of blocks meeting $Y$. So $Y \subset \widehat{Y}$. As usual, define $\partial_{h} \widehat{Y}=$ $\widehat{Y} \cap H$. The subset $\widehat{Y}$ is not a submanifold of $M$ but it does have the structure of an $I$-bundle. So let $\widehat{F}$ be a section of $\widehat{Y}$ which contains $F$ and which is disjoint from $\partial_{h} \widehat{Y}$. 


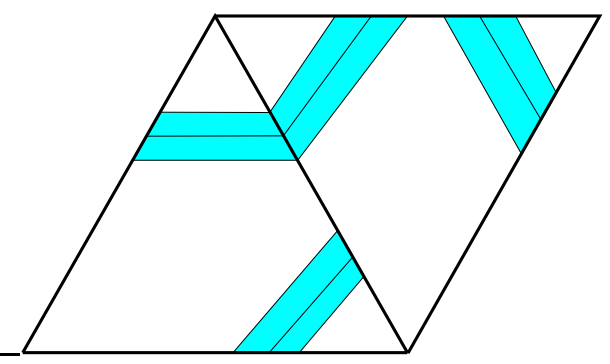

Figure 11: A bit of $\Theta_{F}$ with the nearby $\Theta_{Y}$ shaded

Define $\Theta_{Y}=\partial_{v} \widehat{Y}=\overline{\operatorname{fr}_{M}(\widehat{Y}) \backslash \partial_{h} \widehat{Y}}$. This is a regular neighborhood, taken in $T_{M}^{2}$, of the graph $\Theta_{F}=\operatorname{fr}_{M}(\widehat{F})$ which is also contained in $T_{M}^{2}$. (See Figure 11)

Before continuing on we will tidy up the graph $\Theta_{F}$ a bit. Recall that every tetrahedron of $T_{M}$ is modelled on the regular Euclidean tetrahedron of sidelength one. Apply a normal isotopy of $M$ (i.e. an isotopy fixing every simplex of $T_{M}$ setwise) to make every normal disk of $\widehat{F}$ meeting $\Theta_{F}$ straight. That is, after the isotopy, every normal triangle of $\widehat{F}$ which meets $\Theta_{F}$ is a geodesic triangle in its containing tetrahedron while every quad is either a geodesic quadrilateral or a union of two geodesic triangles. We also require that every edge of $\Theta_{F}$ lies outside of a 1/3-neighborhood of the vertices of $T_{M}^{0}$.

Our next goal will be to bound the complexity of the graph $\Theta_{F}$.

\subsection{Bounding the Complexity of $\Theta_{F}$}

Claim $\Theta_{F}$ has at most $4 t$ edges and at most $4 t$ vertices.

Proof Every edge of $\Theta_{F}$ lies inside a vertical rectangle of $\Theta_{Y}$ and each of these is simultaneously a vertical rectangle of some product block of $\widehat{Y}$ and of some core block of $V$. Thus to bound the edges of $\Theta_{F}$ it suffices to bound the number of vertical rectangles appearing in the boundaries of core blocks.

Recall that the splitting $H$ does not contain an almost normal annulus, by Claim 6.7. Thus each core block in $V$, disjoint from the almost normal octagon, meets $\widehat{Y}$ along at most two vertical rectangular faces. The core block which contains the almost normal octagon, if it exists, meets $\widehat{Y}$ in at most four rectangular faces. Also, at most $2 t$ of the core blocks in $V$ have a vertical rectangle as a face. (See Figure [7) 
Now, the tetrahedron containing the octagon contains only one core block with vertical rectangles. Deduce that $\Theta_{Y}=\partial_{v} \widehat{Y}$ is a union of at most $4 t$ rectangles lying in the two-skeleton. It follows that the number of edges of $\Theta_{F}$ is at most $4 t$. We now must bound the number of vertices in $\Theta_{F}$.

Consider a vertex $v$ of $\Theta_{F}^{0}$. As $\Theta_{F}$ is a union of edges, the vertex $v$ has valence one or higher. Suppose $v$ has valence one exactly. Now, $v$ sits on some edge, $e$, of the triangulation $T_{M}^{1}$ and $e$ intersects exactly one vertical rectangle, $E$, of $\Theta_{Y}$. Thus in every tetrahedron containing $e$ there is a product block of $\widehat{Y}$ in that tetrahedron containing $v$. It follows that $E$ intersects the interior of $Y$, a contradiction. It follows that $v$ must have valence two or higher. Thus $\Theta_{F}$ has at most $4 t$ vertices.

\subsection{Triangulating $\partial R$}

We will now triangulate $\partial R=\partial N$ as well as a "crust", $\Delta_{F}$, which connects $\Theta_{F}$ to a collection of longitudes for $R$. This will permit an efficient triangulation of $N$.

Let $\Delta_{F}=\overline{\widehat{F} \backslash(R \cup F)}$. Then the frontier of $\Delta_{F}$ is the union of $\Theta_{F}$ and a collection of longitudes, $\left\{\lambda_{i}\right\}$, for the tori $R_{i} \subset R$. See Figure 12

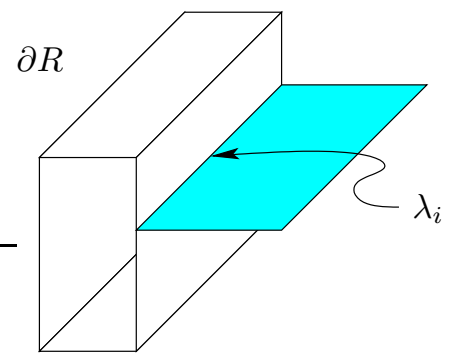

Figure 12: A cross-sectional view of $\partial R\left(\Delta_{F}\right.$ shaded $)$

Choose $\epsilon>0$ very small so that $\eta_{N}\left(\Theta_{F}, 5 \epsilon\right)$, the $5 \epsilon$ neighborhood of $\Theta_{F}$, is a regular neighborhood, and so that every edge of $\Theta_{F}$ has length at least $100 \epsilon$. Perform another normal isotopy of $M$, fixing $\widehat{F}$ setwise, so that the longitudes $\lambda_{i}=\Delta_{F} \cap \partial R_{i}$ lie on the boundary of the handlebody $\eta_{N}\left(\Theta_{F}, \epsilon\right)$.

Partition each longitude $\lambda_{i}$ into two sets of arcs as follows. First for every open tetrahedron $\tau_{j} \in T_{M}^{3}$ there is a collection of $\operatorname{arcs}\left\{\alpha_{i}^{j, k}\right\}=\lambda_{i} \cap \eta\left(\tau_{j}^{1}, 2 \epsilon\right)$. Here $\tau_{j}^{1}$ is the one-skeleton of the tetrahedron $\tau_{j}$ while $j$ runs from 1 to $\left|T_{M}^{3}\right|$. The 
$\operatorname{arcs}\left\{\beta_{i}^{j, k}\right\}$ are the components of $\left.\overline{\lambda_{i} \backslash\left(\cup_{j, k} \alpha_{i}^{j, k}\right.}\right)$. Count the number of $\beta$ 's and $\alpha$ 's:

Claim The number of $\beta$ 's is at most $4 t$ while the number of $\alpha$ 's is at most $24 t^{2}$.

Proof For every edge $e \in \Theta_{F}$ there is exactly one of the $\beta_{i}^{j, k}$,s lying in the closed $\epsilon$-neighborhood of $e$. This bounds the number of $\beta$ 's.

Fix $v \in \Theta_{F}^{0}$. Suppose that $v \in e$ where now $e$ is an edge of $T_{M}^{1}$, the oneskeleton of $M$. Note that $e$ appears as an edge of triangles in $T_{M}^{2}$ in at most $6 t$ ways (this is a very crude bound). So there are at most $6 t-1$ product blocks in $\widehat{Y}$ containing the vertex $v$. It follows that there are at most $6 t-1$ of the $\alpha_{i}^{j, k}$, s within the $2 \epsilon-$ neighborhood of $v$. It follows that there are at most $4 t \cdot 6 t$ of the $\alpha$ 's in total.

Perform a final normal isotopy fixing $H \cup T_{M}^{2}$ pointwise, fixing the points $\partial \alpha_{i}^{j, k}$ for all $i, j, k$, and sending the $\operatorname{arcs}\left\{\alpha_{i}^{j, k}\right\} \cup\left\{\beta_{i}^{j, k}\right\}$ to Euclidean line segments.

Triangulate $\partial R_{i}$ and $\Delta_{F}$ near $\alpha_{i}^{j, k}$ as shown in Figure 13. Triangulate $\partial R_{i}$ and $\Delta_{F}$ near $\beta_{i}^{j, k}$ as shown in Figure 14. It follows that:

Claim The triangulation of $\partial R$ contains at most $6\left(4 t+24 t^{2}\right)$ triangles. Also, the triangulation of $\Delta_{F}$ contains at most $8 t+24 t^{2}$ triangles. Finally, $\partial F$ meets the one-skeleton of $\partial R$ in at most $2\left(4 t+24 t^{2}\right)$ points.

So the boundary-weight of $F$ in $N$ will be at most $2\left(4 t+24 t^{2}\right)<2^{6} t^{2}$, as desired.

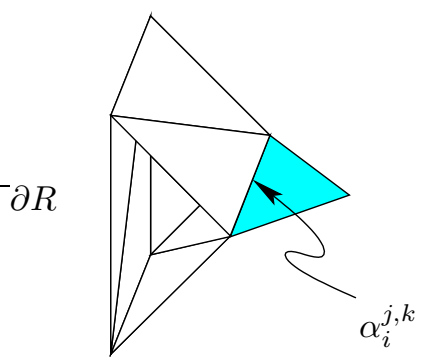

Figure 13: Triangulation of $\partial R$ near $\alpha_{i}^{j, k}\left(\Delta_{F}\right.$ shaded $)$ 


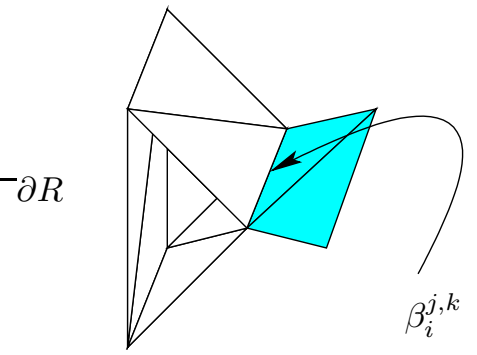

Figure 14: Triangulation of $\partial R$ near $\beta_{i}^{j, k}\left(\Delta_{F}\right.$ shaded $)$

\subsection{Triangulating $N$}

We will now subdivide the balls $\left\{\tau \backslash\left(R \cup \Delta_{F}\right)\right\}$ to obtain a triangulation of $N$.

Every vertex of $T_{M}^{0}$ and $\Theta_{F}^{0}$ is included in the vertex set of $T_{N}$. Fix a face $\sigma \subset T_{M}^{2}$. Let $\delta_{1}, \delta_{2}, \delta_{3}$ be the edges of $\sigma$. Let $\sigma^{\prime}$ be a component of $\sigma \backslash \Theta_{F}$. Then, for each $\sigma^{\prime}$, add a point of the interior of $\sigma^{\prime}$ to the vertex set of $T_{N}$. Also, cone from this point to the boundary of $\sigma^{\prime}$. See Figure [15] Thus $\sigma$ is subdivided into the following number of triangles:

$$
2\left|\sigma \cap \operatorname{interior}\left(\Theta_{F}^{1}\right)\right|+3+\sum_{1=1}^{3}\left|\delta_{i} \cap \Theta_{F}^{0}\right| .
$$

For each tetrahedron $\tau \subset T_{M}^{3}$ let $\tau^{\prime}$ be the closure of $\tau \backslash\left(R \cup \eta\left(\Delta_{F}\right)\right)$. Then $\tau^{\prime}$

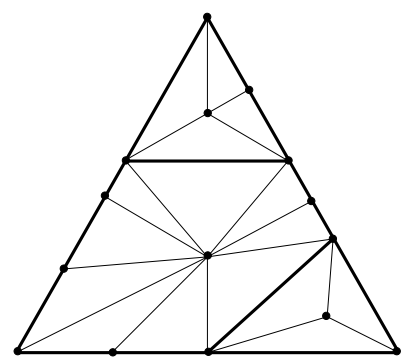

Figure 15: The subdivision of a face $\sigma$

is homeomorphic to a round ball and the chosen triangulation of $T_{M}^{2} \cup \Delta_{F} \cup \partial R$ induces a triangulation on the boundary of $\tau^{\prime}$. To finish, choose a point in the interior of $\tau^{\prime}$ and cone from this point to the boundary of $\tau^{\prime}$. Doing this for every tetrahedron $\tau \subset T_{M}^{3}$ gives the desired triangulation, $T_{N}$. Note that each 
triangle of the subdivided $\sigma \subset T_{M}^{2}$ gives us two tetrahedra in $T_{N}$ as does every triangle in the $\Delta_{F}$. However, each triangle of $\partial R$ gives us only one tetrahedron in $T_{N}$. Adding, find that $\left|T_{N}^{3}\right|$ is at most

$$
2 \sum_{\sigma}\left(2\left|\sigma \cap \operatorname{interior}\left(\Theta_{F}^{1}\right)\right|+3+\sum_{1=1}^{3}\left|\delta_{i} \cap \Theta_{F}^{0}\right|\right)+2\left(8 t+24 t^{2}\right)+6\left(4 t+24 t^{2}\right) .
$$

The above quantity is at most:

$$
\begin{gathered}
16 t+12 t+2(6 t \cdot 4 t)+16 t+48 t^{2}+24 t+144 t^{2} \\
=68 t+240 t^{2}<2^{9} t^{2} .
\end{gathered}
$$

Here $t=\left|T_{M}^{3}\right|$ is the number of tetrahedra in $T_{M}$. Deduce that $N$ admits a triangulation, $T_{N}$, with $\left|T_{N}^{3}\right|<2^{9} t^{2}$ where $F$ has boundary-weight $w(\partial F)<$ $2^{6} t^{2}$. This completes the proof of Claim 9.1

\subsection{Normalizing the middle surface, $F$}

Claim 9.2 There is a proper isotopy $\mathcal{F}: F \times I \rightarrow N$ such that $F^{\prime}=\mathcal{F}_{1}(F)$ is normal with respect to $T_{N}, w\left(F^{\prime}\right) \leq w(F), w\left(\partial F^{\prime}\right) \leq w(\partial F)$.

All weights here are measured with respect to the triangulation $T_{N}$.

Proof Recall that $F$ is incompressible and boundary-incompressible and that $N$ is irreducible and boundary-irreducible. Thus Haken's normalization procedure (Lemma 3.1 in this paper) gives the desired isotopy.

Make the further assumption that $F^{\prime}$, as given by Claim 9.2 minimizes the lexicographic complexity $\left(w\left(\partial F^{\prime}\right), w\left(F^{\prime}\right)\right)$. Abusing notation slightly again use $F$ to denote the normal surface $F^{\prime}$. Decompose $F$ as a sum of fundamental surfaces of the triangulation $T_{N}$ :

$$
F=\sum_{i} m_{i} G_{i}+\sum_{i} n_{i} F_{i}+\sum_{i} p_{i} T_{i}
$$

Here the $G_{i}$ are fundamental surfaces with nonempty boundary, the $F_{i}$ are closed fundamental surfaces with Euler characteristic negative, and the $T_{i}$ are also closed, fundamental, but with Euler characteristic non-negative. Note that, by Claim 9.1 and the lexicographic minimality of $F$, the sum $\sum m_{i}$ is bounded above by $2^{6} t^{2}$. This is because each $G_{i}$ has non-trivial boundary and boundary-weight is additive under Haken sum. 
Euler characteristic is also additive under Haken sum. Thus

$$
\begin{aligned}
& \chi\left(\sum m_{i} G_{i}\right)>-2^{6} t^{2} \cdot \exp \left(14\left|T_{N}^{3}\right|\right) \geq \\
& \geq-2^{6} t^{2} \cdot \exp \left(14 \cdot 2^{9} t^{2}\right)>-\exp \left(2^{14} t^{2}\right) .
\end{aligned}
$$

The first inequality follows from Lemma 3.4. Recall that the Euler characteristic of $F$ is less than $-\exp \left(2^{15} t^{2}\right)$. Hence some coefficient $n_{i}$ is non-zero, say $n_{1} \geq 1$. Rewrite the Haken sum in the form $F=J+K$ where $K=F_{1}$. The surface $J$ is then the sum of all the other terms. Note that $\partial K=\emptyset$.

Properly isotope $J$ and $K$ to obtain the reduced sum $F=J^{\prime}+K^{\prime}$, as defined in Section 3. Abuse notation again to write $F=J+K$.

\section{The final annulus}

Here we analyze the patches, seams, and exchange bands of the reduced sum $F=J+K$. The exchange bands of this sum are our second source of essential annuli and Möbius bands. It is one of these which yields the final contradiction.

Claim 10.1 Every seam of $J+K$ is a closed regular neighborhood of an essential simple closed curve in F. Every exchange band is an annulus or Möbius band. Also, there is at least one seam which is not boundary-parallel in $F$.

Proof The first two claims follows from Lemma 3.5 and from the fact that $\partial K=\emptyset$.

To prove the third proceed by contradiction. That is, suppose every patch but at most one is an annulus. As $\chi(K)<0$ deduce that $J$ is a union of annuli. Thus $\chi(J)=0$. It follows that $\chi(F)=\chi(K)=\chi\left(F_{1}\right)$ which is impossible.

\subsection{Examining the exchange band $A$}

Fix a seam of $J+K$ which is not boundary-parallel inside of $F$. Let $A$ be the exchange band meeting this seam. The argument finishes with a discussion of this exchange band, $A$. As above, the hypothesis that $H$ is full rules out several possibilities. The eventual conclusion will be that the splitting $H$ admits a joined annulus/annulus or Möbius/annulus pair. This implies that the splitting has the DCP and yields the desired contradiction. 
Let $\partial_{-} A \subset \partial A$ be the component of $\partial A$ meeting the seam which is not boundary-parallel in $F$. Let $B=\pi^{-1}\left(\partial_{-} A\right)$ be the vertical surface which is the union of all fibres meeting $\partial_{-} A$. Recall, from Remark 3.2 that all seams are orientation-preserving in $F$. Thus, $B$ is in fact an annulus.

Claim 10.2 The annulus $B$ is essential in $V$.

Proof Suppose that $B$ compresses in $V$. Then, by Claim 7.4 the components of $\partial B$ bound disks in $\partial_{h} Y$. Thus $\partial_{-} A=\pi(\partial B)$ is null-homotopic in $F$, contradicting Claim 10.1

Suppose that $B$ is boundary-parallel in $V$. Let $C \subset H$ be the annulus cobounded by $\partial B$. Let $X \subset V$ be the solid torus bounded by $B \cup C$. Note that $X$ admits a meridional disk meeting each of $B$ and $C$ in a single arc.

If $C \subset \partial_{h} Y$ then $X \subset Y$ and $X$ is the union of the fibres of $Y$ meeting $C$, as $B$ is vertical. Thus $X$ is an $I$-bundle over a Möbius band and any meridional disk meets $B$ in at least two arcs, a contradiction. So $C$ is not contained in $\partial_{h} Y$. Thus $C$ contains some components of $\partial\left(\partial_{h} Y\right)$. By Claim 7.2 every such is a core curve for $C$. It follows that $\partial B$ is boundary-parallel in $\partial_{h} Y$ and thus $\partial_{-} A \subset B$ is boundary-parallel in $F$. This contradicts the choice of exchange band $A$.

\subsection{Constructing the annulus $A^{\prime}$}

As $Y$ is an $I$-bundle there is a small isotopy of $Y$ to $Y^{\prime}$ fixing $F \cup \partial N$ pointwise so that after the isotopy $A \cap Y^{\prime} \subset\left(\pi^{\prime}\right)^{-1}(\partial A)$. Here $\pi^{\prime}: Y^{\prime} \rightarrow F$ is the obvious bundle map. We will also insist that $A \cap Y^{\prime}=\eta_{A}(\partial A)$. Again, abuse notation and refer to $Y^{\prime}$ and $\pi^{\prime}$ as $Y$ and $\pi$.

Let $A^{\prime}=A \backslash \operatorname{interior}(Y)$. Label $\partial A^{\prime}$ and $\partial B$ so that $\partial_{-} A^{\prime}=\partial_{+} B=A^{\prime} \cap B$. Isotope $H$, rel $Y \cup R$, to minimize the quantity $\left|H \cap A^{\prime}\right|$. Once more, abuse notation and denote the resulting Heegaard splitting surface by $H$.

Claim 10.3 All curves of $A^{\prime} \cap H$ are essential in $A^{\prime}$.

Proof Suppose $\delta \subset A^{\prime} \cap H$ bounds an innermost disk $D \subset A^{\prime}$. Now $\delta$ must be essential in $H$. (If not $\delta$ bounds a disk $E \subset H$. As usual, because $\partial_{h} Y$ is nonplanar, $E \cap \partial_{h} Y=\emptyset$. So $E \cup D$ forms a two-sphere in $M$ and this bounds a ball, $U$ (Claim 6.1). Since $(Y \cup R) \cap U=\emptyset$ (Claim 8.3) there is an isotopy of $H$, rel $Y \cup R$, which moves $E$ across $U$. This reduces $\left|A^{\prime} \cap H\right|$, an 
impossibility.) Thus $D$ is an essential disk in $V$ or $W$ which is disjoint from $\partial_{h} Y$. By Remark 7.1 the disk $D$ is one-half of a disjoint disk/annulus pair, implying that $H$ has the DCP, a contradiction.

Let $A^{\prime \prime}$ be the closure of the component of $A^{\prime} \backslash H$ such that $A^{\prime \prime}$ contains $\partial_{-} A^{\prime}=$ $\partial_{+} B$. Note that $A^{\prime \prime} \subset W$. Our task is to show that $A^{\prime \prime}$ and $B$ are a joined pair of essential surfaces. Begin as follows:

Claim 10.4 If $A^{\prime \prime}$ is an annulus then $A^{\prime \prime}$ is incompressible in $W$.

Proof A compression of $A^{\prime \prime}$ would give a properly embedded disk $(D, \partial D) \subset$ $\left(W, \partial_{+} B\right)$. Recall that $B=\pi^{-1}\left(\partial_{-} A\right)$, is essential in $V$ (Claim 10.2). Thus $D$ and $B$ form a joined disk/annulus pair which implies that $H$ is weakly reducible.

Claim 10.5 The annulus or Möbius band $A^{\prime \prime}$ is essential in $W$.

This will complete the proof of Proposition 5.1 as $A^{\prime \prime}$ and $B$ together form an joined annulus/annulus or Möbius/annulus pair. This last shows that $H$ has the DCP and thus could not have been a full Heegaard splitting.

Proof of Claim 10.5 There is a penultimate dichotomy left to consider - If $A^{\prime \prime}$ is strictly contained in $A^{\prime}$ then the situation is fairly simple. If $A^{\prime \prime}=A^{\prime}$ then a slightly more subtle analysis, using Remark [3.6] obtains.

Suppose that interior $\left(A^{\prime}\right) \cap H \neq \emptyset$. That is, $A^{\prime \prime} \neq A^{\prime}$. Then $A^{\prime \prime}$ is an annulus and hence incompressible in $W$ by Claim 10.4. Suppose that $A^{\prime \prime}$ is boundaryparallel. Thus $\partial_{+} B=\partial_{-} A^{\prime \prime}$ is parallel in $H$ to the curve $\partial_{+} A^{\prime \prime} \subset\left(H \backslash \partial_{h} Y\right)$. So $\partial_{+} B$ is boundary-parallel in the surface $\partial_{h} Y$ and thus $\partial_{-} A \subset B$ is boundaryparallel in $F$. This contradicts the choice of seam made in Claim 10.1.

Suppose instead that $A^{\prime} \cap H=\partial A^{\prime}$. Then $A^{\prime \prime}=A^{\prime} \subset W$. There are two final cases: Either $A$ was an annulus or a Möbius band. Suppose that $A$ (and hence $A^{\prime}$ ) is a Möbius band. Now, $A^{\prime}$ is properly embedded in the handlebody $W$ and $W$ is not a solid torus. Thus $A^{\prime}=A^{\prime \prime}$ is essential, as desired. (See Remark [2.2.)

At the last there is the possibility that $A$ is an annulus. Recall $Y$ has been isotoped slightly so that $A^{\prime}=\overline{A \backslash Y}$ and $A^{\prime} \subset W$. Again, $A^{\prime \prime}=A^{\prime}$ is an annulus and hence incompressible in $W$ by Claim 10.4. Suppose $A^{\prime}$ is boundary-parallel in $W$. Let $C \subset H$ be the annulus cobounded by the two components of $\partial A^{\prime}$. 
Let $X \subset W$ be the solid torus bounded by $A^{\prime} \cup C$. If $C$ is not contained in $\partial_{h} Y$ then, as above, deduce that $\partial_{+} B$ is boundary-parallel in $\partial_{h} Y$. This contradicts the choice of seam made in Claim [10.1. Conclude instead that $C \subset \partial_{h} Y$.

It follows $A$ is parallel, relative to $\partial A$ and through $X$, into the surface $F$. As observed in Remark 3.6 this gives a contradiction - for the reader's convenience here are the details: Let $C^{\prime} \subset F$ be the annulus cobounded by the two boundary components of $A$ and let $X^{\prime}$ be the solid torus bounded by $A \cup C^{\prime}$. Let $Z$ be the closure of $\eta_{M \backslash F}(A)$. Let $\widetilde{A}$ be the closure of $\partial Z \backslash F$. Finally, let $F^{\prime}$ be a slight push-off of $(F \backslash Z) \cup \widetilde{A}$. In the terminology of Jaco and Oertel [7] the surface $F^{\prime}$ is obtained by performing an irregular exchange along the exchange annulus $A$. Note that $F^{\prime}$ is the union of a torus and a surface, $F^{\prime \prime}$, isotopic to $F$. For a schematic cross-sectional view see Figure 16.

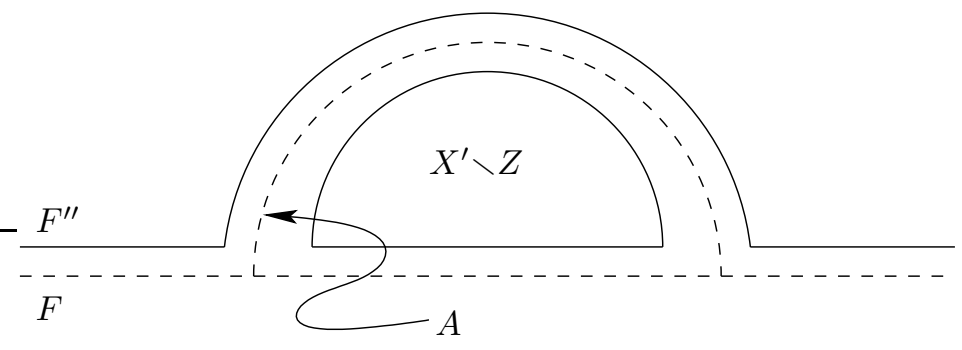

Figure 16: An irregular exchange along $A$

Either $w\left(F^{\prime \prime}\right)<w(F)$ or $F^{\prime \prime}$ is not normal (consult Figure 4). In the latter case there is a weight reducing isotopy of $F^{\prime \prime}$ as in Lemma 3.1 (See Figure 3 with $F^{\prime \prime}$ replacing $F$.) In either case find a contradiction to the assumed lexicographic minimality of $(w(\partial F), w(F))$. It follows that $A^{\prime}$ was not boundary-parallel in $W$. Hence $A^{\prime}=A^{\prime \prime}$ is essential in $W$.

\section{Deducing the main theorem}

Proposition 5.1 is cast into the realm of general splittings using Theorem 3.7

Theorem 11.1 Fix $M$, a closed, orientable three-manifold. There is a constant $C(M)$ such that if $H \subset M$ is a Heegaard splitting, with $g(H)>C(M)$, then $H$ has the disjoint curve property.

Proof Let $T_{M}$ be a minimal triangulation of $M$ and take $C(M)=\exp \left(2^{16} t^{2}\right)$, where $t=\left|T_{M}^{3}\right|$. Suppose that $g(H)$ is at least this big. If $H$ is strongly 
irreducible then, by Theorem 3.7 isotope $H$ to be almost normal with respect to $T_{M}$. Thus $H$ has the DCP by Proposition 5.1. On the other hand, if $H$ is weakly reducible then $H$ trivially satisfies the DCP.

\section{Conjecture}

Recall the generalized Waldhausen conjecture states that:

Conjecture 1.1 If $M$ is closed, orientable, and atoroidal then $M$ contains only finitely many strongly irreducible splittings, up to isotopy, in each genus.

Now fix attention on a closed orientable manifold $M$. If $M$ is a lens space then all splittings are stabilizations of the genus one splitting [1, and hence all splittings other than the genus one splitting have the DCP. We thus restrict ourselves to manifolds with Heegaard genus two or larger. If $M$ is toroidal then by Thompson's Lemma 2.7 all splittings in $M$ have the DCP. If $M$ is atoroidal then, by Theorem 11.1 above a certain genus all splittings have the DCP and, by Conjecture 1.16 , there are only finitely many splittings up to isotopy below that genus. So it would follow that:

Conjecture 12.1 In any closed, orientable three-manifold there are only finitely many full Heegaard splittings, up to isotopy.

In addition to Conjecture 12.1 affirmative answers to any of the following questions would be very interesting.

Question Suppose $M$ is closed, orientable and $M$ admits a full Heegaard splitting. By Lemmas 2.3, 2.7 and2.8 it follows that $M$ is irreducible, atoroidal, and not a Seifert fibred space. So from Thurston's geometrization conjecture it would follow that $M$ is hyperbolic. Weaker, but still extremely interesting, would be a direct proof that $M$ had word-hyperbolic, infinite, or simply nontrivial fundamental group.

Note that the converse statement, that every hyperbolic manifold contains a full splitting, cannot be obtained. It follows from work of Rieck and Sedgwick [15, combined with that of Kobayashi [12, that for "most" surgeries on two-bridge knots the manifold obtained does not admit a full splitting. This answers a question of Luo's in the negative: There are closed hyperbolic three-manifolds which do not admit a "hyperbolic" splitting. (See Section 5 of [13].) 
More directly concerned with the work of this paper would be an improvement of the bound given in Theorem 11.1. A "yes" answer to the following question would be very satisfying:

Question Do full Heegaard splittings always have minimal genus?

In light of the above, Sedgwick has asked a modified version of Waldhausen's conjecture:

Question If $M$ is non-Haken, does $M$ contain only finitely many non-isotopic, irreducible Heegaard splittings?

Establishing this result would nicely complement Conjecture 12.1 as well as reaffirm the Casson-Gordon program of studying non-Haken three-manifolds via their strongly irreducible Heegaard splittings.

\section{References}

[1] Francis Bonahon, Jean-Pierre Otal, Scindements de Heegaard des espaces lenticulaires, Ann. Sci. École Norm. Sup. (4) 16 (1983) 451-466 (1984)

[2] Andrew J Casson, Cameron McA Gordon, Reducing Heegaard splittings, Topology Appl. 27 (1987) 275-283

[3] Wolfgang Haken, Some results on surfaces in 3-manifolds, from: "Studies in Modern Topology", Math. Assoc. Amer. (distributed by Prentice-Hall, Englewood Cliffs, N.J.) (1968) 39-98

[4] Joel Hass, Jeffrey C Lagarias, Nicholas Pippenger, The computational complexity of knot and link problems, J. ACM 46 (1999) 185-211

[5] John Hempel, 3-manifolds as viewed from the curve complex, Topology 40 (2001) 631-657

[6] William Jaco Personal communication

[7] William Jaco, Ulrich Oertel, An algorithm to decide if a 3-manifold is a Haken manifold, Topology 23 (1984) 195-209

[8] William Jaco, Jeffrey L Tollefson, Algorithms for the complete decomposition of a closed 3-manifold, Illinois J. Math. 39 (1995) 358-406

[9] Tsuyoshi Kobayashi, Casson-Gordon's rectangle condition of Heegaard diagrams and incompressible tori in 3-manifolds, Osaka J. Math. 25 (1988) 553-573

[10] Tsuyoshi Kobayashi, Heights of simple loops and pseudo-Anosov homeomorphisms, from: "Braids (Santa Cruz, CA, 1986)", Amer. Math. Soc., Providence, RI (1988) 327-338 
[11] Tsuyoshi Kobayashi, A construction of 3-manifolds whose homeomorphism classes of Heegaard splittings have polynomial growth, Osaka J. Math. 29 (1992) 653-674

[12] Tsuyoshi Kobayashi, Heegaard splittings of exteriors of two bridge knots, Geom. Topol. 5 (2001) 609-650 (electronic)

[13] Feng Luo, Heegaard diagrams and handlebody groups, Topology Appl. 129 (2003) 111-127

[14] Yoav Moriah, Jennifer Schultens, Irreducible Heegaard splittings of Seifert fibered spaces are either vertical or horizontal, Topology 37 (1998) 1089-1112

[15] Yo'av Rieck, Eric Sedgwick, Finiteness results for Heegaard surfaces in surgered manifolds, Comm. Anal. Geom. 9 (2001) 351-367

[16] J Hyam Rubinstein, Polyhedral minimal surfaces, Heegaard splittings and decision problems for 3-dimensional manifolds, from: "Geometric topology (Athens, GA, 1993)", Amer. Math. Soc., Providence, RI (1997) 1-20

[17] Martin Scharlemann, Local detection of strongly irreducible Heegaard splittings, Topology Appl. 90 (1998) 135-147

[18] Martin Scharlemann, Heegaard splittings of compact 3-manifolds, from: "Handbook of geometric topology", North-Holland, Amsterdam (2002) 921953, arXiv:math.GT/0007144

[19] Eric Sedgwick, An infinite collection of Heegaard splittings that are equivalent after one stabilization, Math. Ann. 308 (1997) 65-72

[20] Michelle Stocking, Almost normal surfaces in 3-manifolds, Trans. Amer. Math. Soc. 352 (2000) 171-207

[21] Abigail Thompson, The disjoint curve property and genus 2 manifolds, Topology Appl. 97 (1999) 273-279

[22] Friedhelm Waldhausen, Heegaard-Zerlegungen der 3-Sphäre, Topology 7 (1968) 195-203 


\section{Appendices}

\section{A Surfaces}

Recall that the complexity of a compact surface $G$ is defined to be the quantity $c(G)=$ $\chi(G)+|\partial G|$. This is the same as the Euler characteristic of the surface obtained by capping off all boundary components of $G$ by disks. The lemma below essentially states that Euler characteristic increases when a surface is compressed.

Lemma A.1 Suppose that $G$ and $F$ are compact, connected surfaces with $G \subset F$. Then $c(F) \leq c(G)$.

Proof If $G=F$ then the statement is trivial. Assume then that $\partial G \neq \emptyset$ and that $G$ has been isotoped so that $\partial G \cap \partial F=\emptyset$.

Let $G^{\prime}=\bigcup_{i=1}^{n} G_{i}=F \backslash \operatorname{interior}(G)$, where each $G_{i}$ is connected. It follows that $n \leq|\partial G|$ and that $\left|\partial G^{\prime}\right|=|\partial G|+|\partial F|$. As $c(S) \leq 2$ for any compact, connected surface $S$ we have:

$$
c\left(G^{\prime}\right)=\sum c\left(G_{i}\right) \leq 2 n \leq 2|\partial G|
$$

Omitting the middle yields:

$$
\chi\left(G^{\prime}\right)+\left|\partial G^{\prime}\right| \leq 2|\partial G|
$$

Thus:

$$
\chi\left(G^{\prime}\right)+|\partial F| \leq|\partial G| .
$$

Add $\chi(G)$ to both sides to obtain the desired inequality.

\section{B I-bundles}

As a matter of terminology, a subset of an $I$-bundle is called vertical if it is a union of fibres. The next lemma supplies us with many essential annuli inside of handlebodies.

Lemma B.1 Let $Y$ be an $I$-bundle embedded in a handlebody $V$ such that $\partial_{h} Y \subset$ $\partial V$. Let $A$ be a properly embedded vertical annulus of $Y$. Suppose that $A$ is nonseparating inside of $Y$. Then $A$ is an essential annulus in $V$.

Proof As $A$ is nonseparating in $Y, A$ is nonseparating in $V$. Thus $A$ is not boundary-parallel. It is left to show that $A$ is incompressible.

Pick $B$, a vertical annulus or Möbius band in $Y$, such that $\alpha=A \cap B$ is a single fibre. For a contradiction suppose that $D \subset V$ is a compressing disk for $A$. As $\partial D$ is isotopic to the core curve of $A,|\alpha \cap \partial D|$ is odd.

However, by general position, $D \cap B$ is a compact one-manifold with boundary $\alpha \cap \partial D$. But compact one-manifolds have an even number of points on their boundary. Thus $A$ is incompressible. 